\title{
Implementation and validation of a model of the MPI Stewart platform
}

\author{
F.M. Nieuwenhuizen, ${ }^{*}$ \\ Max Planck Institute for Biological Cybernetics, Tübingen, Germany \\ M.M. van Paassen, ${ }^{\dagger}$ M. Mulder, ${ }^{\ddagger}$ \\ Delft University of Technology, Delft, The Netherlands \\ and H.H. Bülthoff ${ }^{\S}$ \\ Max Planck Institute for Biological Cybernetics, Tübingen, Germany \\ Department of Brain and Cognitive Engineering, Korea University, Seoul, South Korea
}

\begin{abstract}
A simulated model of the MPI Stewart platform can be used to identify the influence of motion system characteristics on human control behaviour in active closed-loop control experiments on the SIMONA Research Simulator. In this paper, a previously identified model of the MPI Stewart platform was analysed with describing function measurements, and it was found that a reduced form of the model was sufficient to capture the relevant dynamics. The reduced model was simulated on the SIMONA Research Simulator and describing function measurements were performed with two IMUs. Both IMUs revealed a resonance peak in measurements of response magnitude at the highest frequencies. A reduced time delay was found with the newer IMU. With the describing function measurements, the implementation of the MPI Stewart platform model was validated in terms of the frequency response and the time delay.
\end{abstract}

\section{Introduction}

Full flight simulators are used for pilot training throughout the world and provide an effective, efficient, and safe environment for practicing flight-critical manoeuvres outside the real aircraft. However, there is an on-going debate about the effectiveness of using simulator motion systems. There is no consensus about the need of simulator motion cueing for pilot training. ${ }^{1}$ Additional indications exist that training without motion may improve pilot-vehicle performance at quasi-transfer to the simulator with motion compared to training in a simulator with motion. ${ }^{2}$ However, in several experiments an increase in performance was found with simulator motion, and an influence on identified parameters of a pilot model, indicating changes in pilot behaviour in the closed-loop control tasks that were performed..$^{3-5}$

The ongoing debate on simulator motion cueing shows that there is a need for further investigations into the influence of simulator motion on pilot control behaviour. Questions arise as to what role motion system characteristics such as platform dynamics play in closed-loop control tasks. Previous research has focussed on the role of motion as complementary cues to, e.g., visual cues, ${ }^{6-8}$ but the influence of motion characteristics has not yet been investigated.

*Ph.D. student, Max Planck Institute for Biological Cybernetics, P.O. Box 2169, 72012 Tübingen, Germany; frank.nieuwenhuizen@tuebingen.mpg.de. Student member AIAA.

${ }^{\dagger}$ Associate Professor, Control and Simulation Division, Faculty of Aerospace Engineering, P.O. Box 5058, 2600GB, Delft, The Netherlands; m.m.vanpaassen@tudelft.nl. Member AIAA.

${ }^{\ddagger}$ Professor, Control and Simulation Division, Faculty of Aerospace Engineering, P.O. Box 5058, 2600GB, Delft, The Netherlands; m.mulder@tudelft.nl. Member AIAA.

$\S$ Professor and Director, Max Planck Institute for Biological Cybernetics, P.O. Box 2169, 72012 Tübingen, Germany; heinrich.buelthoff@tuebingen.mpg.de. Adjunct Professor, Department of Brain and Cognitive Engineering, Korea University, Seoul, South Korea. Member AIAA. 
A recent project had the goal of determining the characteristics of the MPI Stewart platform and building a dynamic model of the platform. The MPI Stewart platform is a mid-size hexapod motion platform with electrical actuators. It is used in passive experiments involving integration of visual and vestibular cues for the perception of self-motion and active experiments related to behaviour of humans in closed-loop control tasks.

A model of the MPI Stewart platform would make it possible to simulate its behaviour on the SIMONA Research Simulator (SRS), which features hydraulic actuators and a larger workspace. In such a setup, the motion characteristics of the MPI Stewart platform could be altered systematically, and by modelling the multi-modal human perception and control behaviour in active closed-loop control tasks, ${ }^{9,10}$ the influence of the motion characteristics can be assessed.

Previously, the dynamic properties of the MPI Stewart platform were determined with a systematic approach based on the AGARD-144 report. ${ }^{11,12}$ A fixed time delay of $35 \mathrm{~ms}$ was measured, and it was found that the default platform filters implemented by the manufacturer caused a rather restricted operating range. Based on these measurements, a dynamic model was determined and validated with simulations. ${ }^{13}$

In this paper, the implementation of the model of the MPI Stewart platform on the SIMONA Research Simulator (SRS) is discussed. First, the characteristics of both simulators are introduced briefly. Second, the model of the MPI Stewart platform and the estimation of its parameters are summarised. Finally, the validation of the model on the SRS is presented and conclusions are drawn.

\section{Research simulators}

The research simulators at the Max Planck Institute for Biological Cybernetics and Delft University of Technology are used to investigate perception and control behaviour of humans in closed-loop control tasks as well as in open-loop perception experiments. The simulators are shown in Fig. 1.

The motion systems of both simulators are configured as a hexapod, which are capable of carrying relatively large payloads and maintaining high rigidity. Most simulator motion systems, e.g. training simulators for airline pilots, are based on this configuration that was applied to flight simulation by Stewart. ${ }^{14}$

Due to the parallel configuration of the actuators, the workspace of hexapod motion systems is highly dependent on the position of the simulator cabin. The extension capabilities of the actuators in terms of position, rate, and acceleration determine the motion capabilities of the simulator cabin. The mathematical relationship between the actuator and simulator cabin capabilities can be described with the kinematic and dynamic equations, given in Section III.

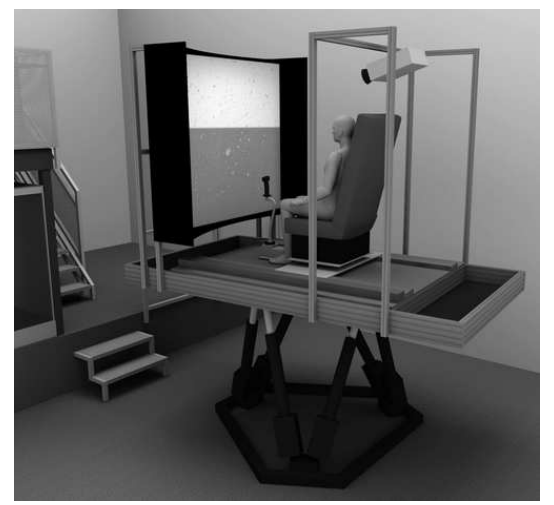

(a) MPI Stewart platform

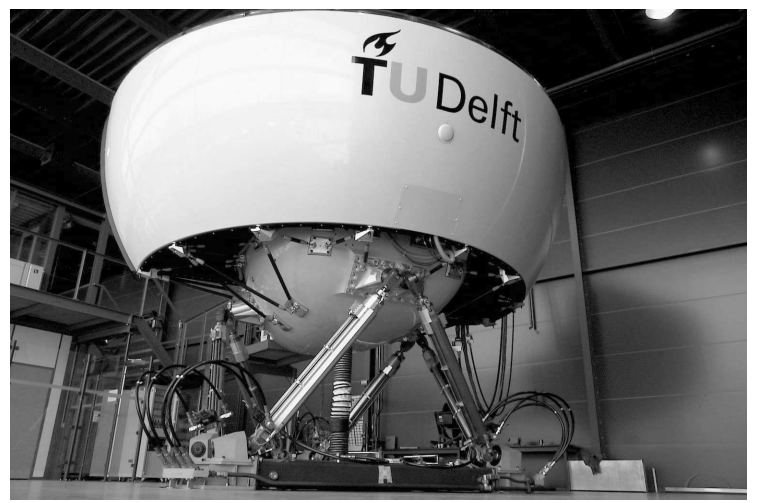

(b) SIMONA Research Simulator

Figure 1: The research simulators at the MPI for Biological Cybernetics and at TUDelft.

The MPI Stewart platform is based on a mid-size motion platform (Maxcue 610-450, MotionBase, United Kingdom). An impression of the MPI Stewart platform is given in Fig. 1a. The platform is equipped with a custom-built cabin that allows for modular adjustments of input devices. A flat or curved screen with a field of view of approximately $72^{\circ}$ horizontally and $53^{\circ}$ vertically can be used as visual display for projections. The platform is controlled through a light-weight in-house software framework that handles the network communication between various computers. 
The motion system of the MPI Stewart platform features platform filters, implemented by the manufacturer, for all degrees of freedom. These filters are not related to motion cueing filters, which scale the output of, e.g., aircraft dynamics such that the simulator stays within its bounds, and which are not used here. A transfer function of the platform filters is given by the following equation:

$$
H_{\text {platform }}=\frac{1}{\left(1+\frac{1}{2 \pi f_{b}} s\right)^{2}},
$$

where $f_{b}$ represents the filter break frequency. Its default value is $1 \mathrm{~Hz}$. Thus, the platform filters are fairly restrictive and reduce the magnitudes of the motion input signals above $1 \mathrm{~Hz}$ drastically. Additionally, the platform filters introduce phase lag that is noticeable during operation of the platform. However, the break frequency can be increased, and values up to $10 \mathrm{~Hz}$ have been used in performance measurements on the MPI Stewart platform. This increases the usable frequency range of the platform considerably.

The SIMONA Research Simulator is depicted in Fig. 1b. The motion system of the SIMONA Research Simulator (SRS) has a similar design to the MPI Stewart platform, but features hydraulic actuators with hydrostatic bearings. ${ }^{15}$ The SRS is equipped with a collimated visual display system with a field of view of $180^{\circ}$ horizontally and $40^{\circ}$ vertically. The cabin features a full generic, 2-person flight deck with control loading devices such as a yoke, sidestick, and pedals. The SRS is controlled through the real-time software framework DUECA developed at TUDelft. ${ }^{16}$

In Table 1, the characteristics of the MPI Stewart platform and the SRS are compared. From the table it is clear that the SRS has a larger workspace and dynamic range. Thus, the SRS can be used to simulate a model of the MPI Stewart platform.

Table 1: Research simulator comparison.

\begin{tabular}{lcc}
\hline \hline & MPI Stewart platform & SRS \\
\hline Range & \\
Surge (mm) & 930 & 2240 \\
Sway $(\mathrm{mm})$ & 860 & 2062 \\
Heave $(\mathrm{mm})$ & 500 & 1314 \\
Roll $(\mathrm{deg})$ & \pm 28 & \pm 25.9 \\
Pitch (deg) & $+34 /-32$ & $+24.3 /-23.7$ \\
Yaw (deg) & \pm 44 & \pm 41.6 \\
\hline Platform filters & & \\
Break frequency $f_{b}$ & Hz (tuneable) & - \\
\hline Actuators & & hydraulic \\
Type & electric & 1.15 \\
Stroke $(\mathrm{m})$ & 0.45 & 13 \\
Max. vel. $(\mathrm{m} / \mathrm{s})$ & 0.3 & \\
Max. acc. $\left(\mathrm{m} / \mathrm{s}^{2}\right)$ & 2 & \\
\hline \hline
\end{tabular}

\section{MPI Stewart platform model}

In previous research, a mathematical model of the MPI Stewart platform was created. ${ }^{13}$ In this section, the model and the estimation of its parameters will be summarised. The final model will be analysed to determine its applicability for simulation on the SRS.

\section{A. Kinematics and dynamics}

The kinematics of a Stewart platform concern the relation between the platform pose (and its derivatives) and the actuator leg lengths, velocities, and accelerations. ${ }^{17-19}$ The platform pose and its derivatives are defined as follows: ${ }^{17}, 18$ 


$$
\bar{x}=\left[\begin{array}{c}
x \\
y \\
z \\
\phi \\
\theta \\
\psi
\end{array}\right], \quad \dot{\bar{x}}=\left[\begin{array}{c}
\dot{\bar{c}} \\
\bar{\omega}
\end{array}\right]=\left[\begin{array}{c}
\dot{x} \\
\dot{y} \\
\dot{z} \\
p \\
q \\
r
\end{array}\right], \quad \ddot{\bar{x}}=\left[\begin{array}{c}
\ddot{\bar{c}} \\
\dot{\bar{\omega}}
\end{array}\right]=\left[\begin{array}{c}
\ddot{x} \\
\ddot{y} \\
\ddot{z} \\
\dot{p} \\
\dot{q} \\
\dot{r}
\end{array}\right] .
$$

Here, the translational degree of freedom in surge is given as $x$, in sway is $y$, and in heave is $z$. The translational degrees of freedom are grouped in vector $\bar{c}$, which is the location of the Upper Gimbal Point (UGP). The platform roll, pitch, and yaw angle are denoted as $\phi, \theta$, and $\psi$, respectively, and have associated angular velocities of the cabin given as $p, q$, and $r$. The angular velocities are grouped in vector $\bar{\omega}$.

With the inverse kinematics, actuator length, velocity, and acceleration can be calculated from the platform pose and its derivatives. As the Stewart platform is a parallel motion system, the inverse kinematics can be calculated analytically. For the inverse position kinematics, the following equation holds: ${ }^{17,18}$

$$
\bar{l}=\bar{c}+T \bar{a}^{c}-\bar{b},
$$

where $\bar{l}$ contains the vectors between the leg attachment points on the base and cabin frame, $T$ is the rotation matrix between the base and cabin frame, and where $\bar{a}$ and $\bar{b}$ are the location matrices of the gimbals of the cabin and base, respectively. The values for the latter two variables are specified by the platform manufacturer and given in Table 2 . The cabin reference frame, denoted by superscript ${ }^{c}$, goes through the UGP, which is centre of the upper frame of the motion system.

Table 2: Gimbal locations of the MPI Stewart platform.

\begin{tabular}{crrrr}
\hline \hline & \multicolumn{2}{c}{ base } & \multicolumn{2}{c}{ cabin } \\
\hline leg & $\mathrm{x}(\mathrm{m})$ & $\mathrm{y}(\mathrm{m})$ & $\mathrm{x}(\mathrm{m})$ & $\mathrm{y}(\mathrm{m})$ \\
\hline 1 & -0.327 & -0.730 & 0.226 & -0.556 \\
2 & 0.796 & -0.082 & 0.369 & -0.473 \\
3 & 0.796 & 0.082 & 0.369 & 0.473 \\
4 & -0.327 & 0.730 & 0.226 & 0.556 \\
5 & -0.469 & 0.648 & -0.594 & 0.082 \\
6 & -0.469 & -0.648 & -0.594 & -0.082 \\
\hline \hline
\end{tabular}

By differentiating Eq. (3), the inverse rate kinematics can be found. These can be written as follows:

$$
\dot{\bar{l}}=J_{l x} \dot{\bar{x}}
$$

where $J_{l x}$ is the platform Jacobian matrix. The columns of $J_{l x}$ specify the velocities required from the actuators to get unit velocity of the platform and its value can be calculated analytically. ${ }^{18}$ In a specific system configuration and pose, the Jacobian is a measure for the kinematic efficiency of the platform motion. It can also be used to derive platform performance measures such as dexterity, manipulability, and stiffness. ${ }^{18}$ Additionally, the inverse acceleration kinematics can be solved. For this, the reader is referred to Ref. 19.

The reverse process to the inverse kinematics is to determine the platform pose from actuator length measurements and is called the forward kinematics. For a general Stewart platform, multiple solutions can be found for the forward kinematics problem. ${ }^{19}$ Thus, the forward kinematics can not be solved analytically and a numerical iterative technique must be applied. In general, a Newton-Raphson method is used to solve the forward kinematic problem. It is formulated as:

$$
\bar{x}_{i+1}=\bar{x}_{i}+\left(J_{l x}\left(\bar{x}_{i}\right)\right)^{-1}\left(\bar{l}_{\text {meas }}-\bar{l}\left(\bar{x}_{i}\right)\right) .
$$

The initial guess $\bar{x}_{0}$ should be sufficiently close to the actual platform pose and could, for example, be the desired platform pose. The iterative process should be repeated until a solution is found with an acceptable tolerance between the measured and calculated actuator lengths. In practical applications, a tolerance level of $10^{-6} \mathrm{~m}$ is reached in $2-3$ iterations. 
The dynamics of the Stewart platform describe the relation between the generalised force/torque vector and the generalised position, velocity and acceleration. The inverse dynamics are used to calculate actuator forces from position and its derivatives. For this, an analytic solution exists, similar to that of the inverse kinematics. The reader is referred to Ref. 19 for more details.

The forward dynamics are used to calculate the motion of the Stewart platform given the actuator forces. When assuming the platform cabin as a rigid body, and disregarding the inertial forces of the actuators, the Stewart platform dynamics can be modelled as follows: ${ }^{17}$

$$
\left[\begin{array}{c}
L_{n} \\
T A^{c} \times L_{n}
\end{array}\right] \bar{f}_{a}=\left[\begin{array}{cc}
m_{c} I & 0 \\
0 & T I_{\bar{c}}^{c} T^{T}
\end{array}\right]\left[\begin{array}{c}
\ddot{\bar{c}} \\
\dot{\bar{\omega}}
\end{array}\right]+\left[\begin{array}{cc}
0 & 0 \\
0 & \Omega T I_{\bar{c}}^{c} T^{T}
\end{array}\right]\left[\begin{array}{c}
\dot{\bar{c}} \\
\bar{\omega}
\end{array}\right]-\left[\begin{array}{c}
m_{c} \bar{g} \\
0
\end{array}\right] .
$$

Here, $L_{n}$ is a matrix that contains the normalised actuator vectors, $A^{c}$ is a matrix that holds the platform gimbal positions in the platform reference frame, $\bar{f}_{a}$ are the actuator forces, $m_{c}$ is the cabin mass, $I$ is the identity matrix, $I_{\bar{c}}^{c}$ is the platform inertia tensor in the cabin reference frame, $\Omega$ is a skew-symmetric matrix that contains the platform angular rates, and $\bar{g}$ is the gravity vector.

A reduced form of the model is given as: ${ }^{17}$

$$
J_{l x}^{T} \bar{f}_{a}=M_{\bar{c}}(\bar{x}) \ddot{\bar{x}}+C_{\bar{c}}(\dot{\bar{x}}, \bar{x}) \dot{\bar{x}}+G_{\bar{c}}
$$

where the influence of the mass matrix $M_{\bar{c}}$, the coriolis and centripetal effects $C_{\bar{c}}$, and the gravity $G_{\bar{c}}$ are clearly separated. The Jacobian $J_{l x}$ is used to transform the actuator forces into the platform coordinate frame.

\section{B. Identification of model parameters}

The dynamic model of a Stewart platform given in Eq. (7) has 10 parameters: the platform mass $m_{c}$, the position of the centre of gravity $x_{\operatorname{cog}}, y_{\operatorname{cog}}, z_{\operatorname{cog}}$, and the values of the inertia tensor $I_{\bar{c}}^{c}$. As the cabin is symmetric in the forward-backward vertical plane, we can assume that the cross products of inertia $I_{x y}=I_{y x}$ and $I_{y z}=I_{z y}$ equal zero. Furthermore, $I_{x z}=I_{z x}$ is assumed to be small with respect to the principal moments $I_{x x}, I_{y y}$, and $I_{z z}$ and therefore negligible. This means that the cabin reference frame is considered as the principle axis of the cabin.

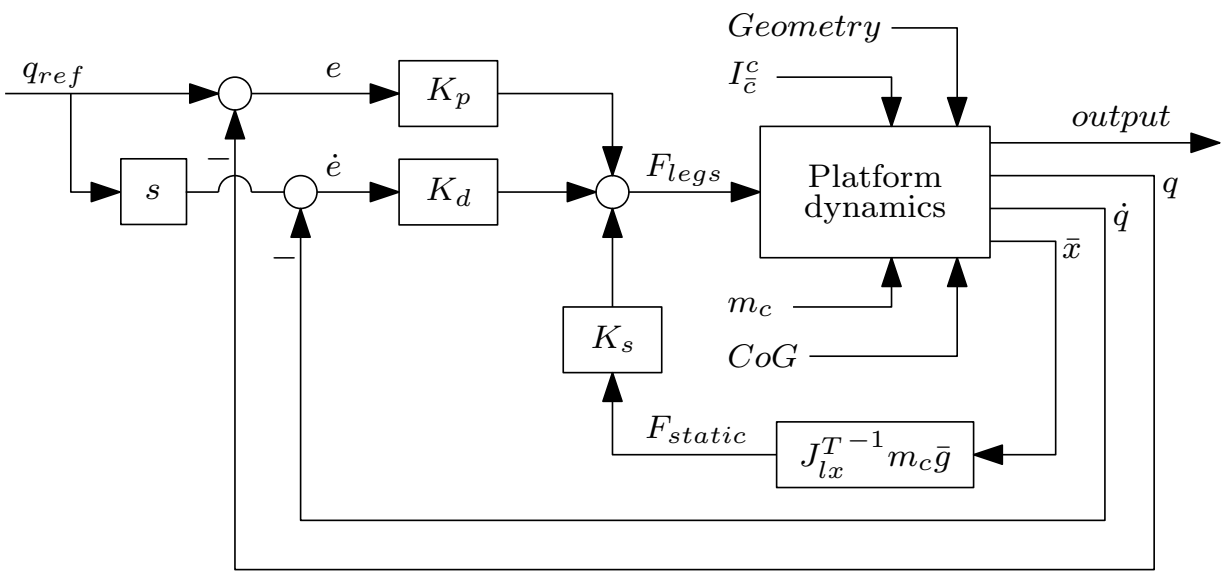

Figure 2: The platform model block diagram.

A block diagram of the complete platform model is given in Fig. 2. Based on the motion system documentation, the controller of the platform has the form of a PD-controller and thus regulates leg lengths and leg velocities. The proportional gain is given as $K_{p}$ and the differential gain as $K_{d}$. The relative contribution of the controller gains is known, but not the exact values. Therefore, $K_{p}$ and $K_{d}$ are expressed in terms of a general controller gain $K_{c}$ by the following expressions:

$$
K_{p}=2 \cdot K_{c} \quad \text { and } \quad K_{d}=7 \cdot K_{c} \text {. }
$$

The controller gain $K_{c}$ is unknown and is estimated in the platform parameter optimisation. 


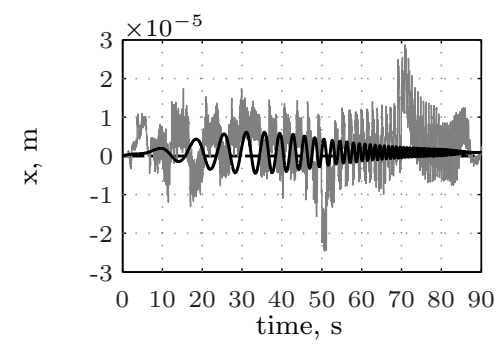

(a) Surge.

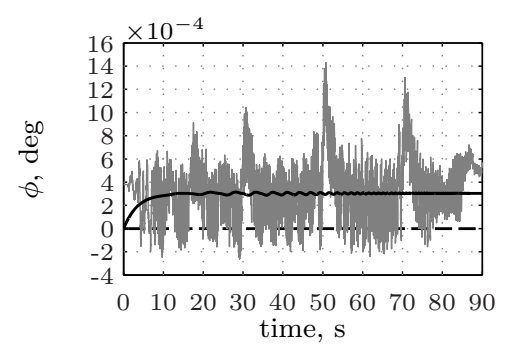

(d) Roll.

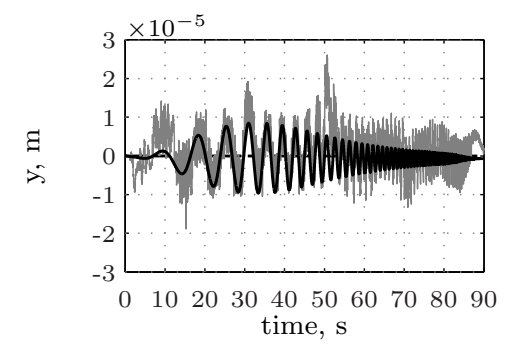

(b) Sway.

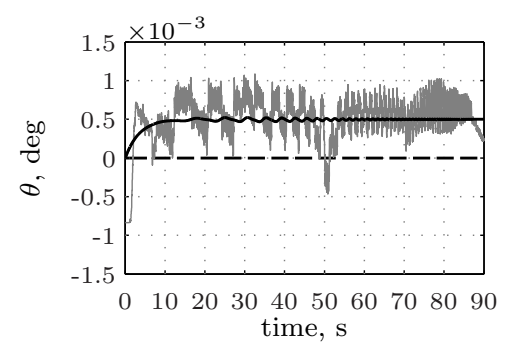

(e) Pitch.

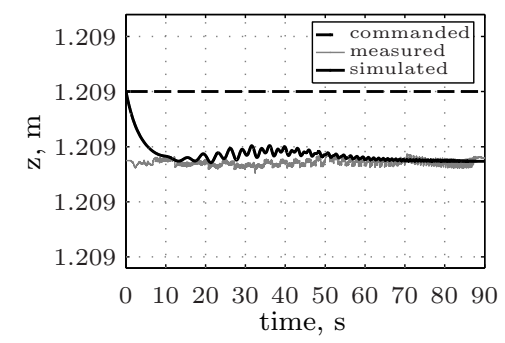

(c) Heave.

Figure 3: Platform commanded, measured and simulated motion for a frequency sweep in yaw. Note the differences in y-axis scaling, the axis for heave spans $230 \mu \mathrm{m}$.

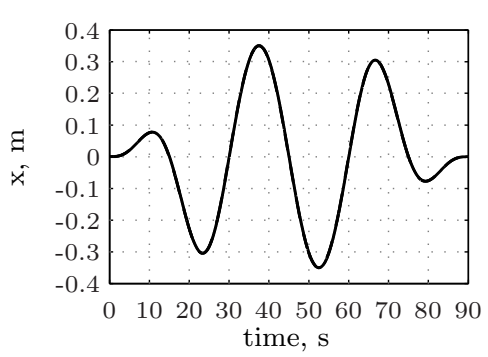

(a) Surge.

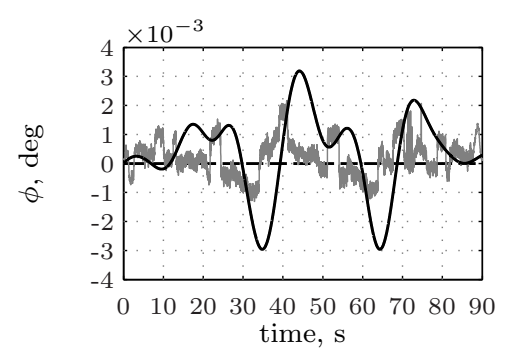

(d) Roll.

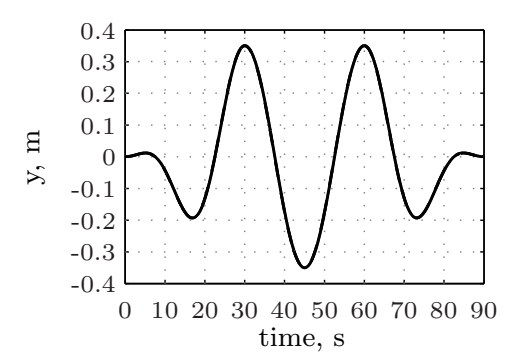

(b) Sway.

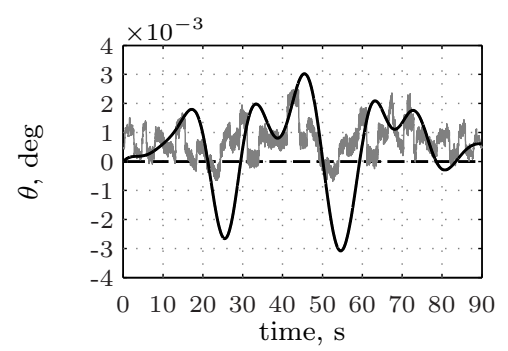

(e) Pitch.

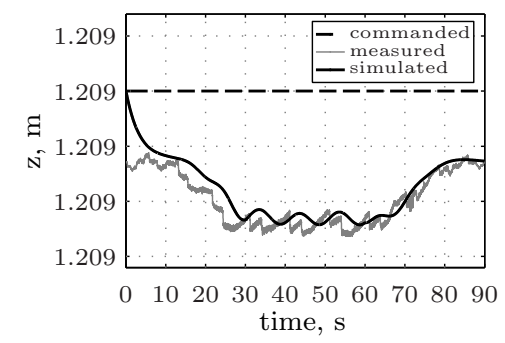

(c) Heave.

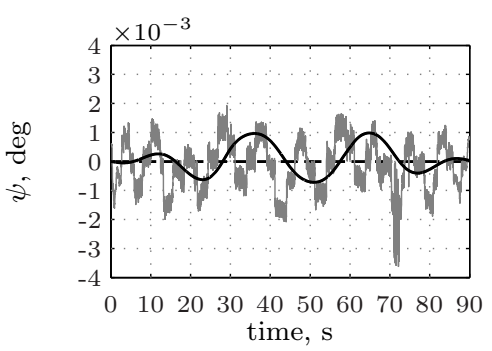

(f) Yaw.

Figure 4: Platform commanded, measured and simulated motion for a circle motion. Note the differences in y-axis scaling, the axis for heave spans $230 \mu \mathrm{m}$. 
The PD-controller of the platform is only responsible for the dynamic platform motion. As can be seen in Fig. 2, a feedback of static forces is implemented based on the current platform position. The gain $K_{s}$ on the feedback of static forces is to account for the bias in leg length found in measurements. In total, nine parameters need to be determined for the dynamic model.

The model parameters were determined by performing frequency sweeps and circular motion measurements on the MPI Stewart platform. The dynamic model was fit to the measured data. Frequency sweeps in the acceleration domain were used to gain insight into the dynamic response of the motion platform. To put more emphasis on the lower frequency range, where the characteristic periods are longer, exponentially increasing sweep frequencies were used. ${ }^{20}$

Results from a frequency sweep in yaw are given in Fig. $3 .^{13}$ It is clear that the model captures the response in the driven axis, as well as in the undriven axes. Measurements in roll and pitch revealed a similar model response in the driven axis, although the response in the undriven axes was slightly too high.

With the frequency sweeps, the platform characteristics were determined around the neutral point, where the actuators are in the middle of their stroke. This is the most important point in the platform workspace, as the largest workspace in all degrees of freedom is available. However, a motion system is intrinsically non-linear and therefore the platform properties might depend on the position within the workspace. Thus, measurements were performed with a circular movement in the horizontal plane.

The commanded, measured, and simulated platform response for the circular motion profile is given in Fig. 4. These measurements show that the platform model can capture the behaviour throughout the workspace well and that the simulated platform position is accurate on a sub-millimetre level. By using a static feedback gain $K_{s}$, the model is capable of tracking the static bias in the measurements in heave, it is clear from Fig. 4c that the controller needs some time to settle.

The final values of the model parameters are given in Table 3. Initial validations of the final model were performed by simulations with independent measurement data that were not used in the model determination process. These simulations showed favourable results with sinusoidal measurements in heave. ${ }^{13}$

Table 3: The estimated platform model parameters.

\begin{tabular}{lcccccccccc}
\hline \hline Parameter & $\begin{array}{c}m_{c} \\
\mathrm{~kg}\end{array}$ & $\begin{array}{c}x_{\operatorname{cog}} \\
\mathrm{m}\end{array}$ & $\begin{array}{c}y_{\operatorname{cog}} \\
\mathrm{m}\end{array}$ & $\begin{array}{c}z_{\operatorname{cog}} \\
\mathrm{m}\end{array}$ & $\begin{array}{c}I_{x x} \\
\mathrm{~kg} \cdot \mathrm{m}^{2}\end{array}$ & $\begin{array}{c}I_{y y} \\
\mathrm{~kg} \cdot \mathrm{m}^{2}\end{array}$ & $\begin{array}{c}I_{z z} \\
\mathrm{~kg} \cdot \mathrm{m}^{2}\end{array}$ & $\begin{array}{c}K_{p} \\
\mathrm{~N} / \mathrm{m}\end{array}$ & $\begin{array}{c}K_{d} \\
\mathrm{~N} \cdot \mathrm{s} / \mathrm{m}\end{array}$ & $\begin{array}{c}K_{s} \\
-\end{array}$ \\
\hline Value & 250 & 0.025 & -0.015 & 0.05 & 825 & 825 & 425 & 21000 & 73500 & 0.9973 \\
\hline \hline
\end{tabular}

\section{Model assumptions}

The dynamic model for the MPI Stewart platform is based on several assumptions. First, the cross products of inertia are assumed zero, i.e., that the platform cabin is symmetric. Second, the gimbal locations are based on specifications by the manufacturer. Small deviations on the MPI Stewart platform are likely and have an impact on the calculated pose and Jacobian matrix. Third, the platform leg measurements are assumed to be properly calibrated by the manufacturer.

Furthermore, the platform cabin is taken as a rigid body and the mass and inertia properties of the actuators are not taken into account. A large relative contribution of the actuator mass to the total mass would warrant inclusion of these effects in the platform model. Finally, hysteresis in the actuators is not modelled.

\section{Validation measurements}

In this section, first the measures are explained that are needed for the validation of the MPI Stewart platform model. After that, the model itself is analysed. Finally, the results from the validation measurements on the SRS are discussed.

\section{A. Describing function measurements}

The validation measurements were performed by determining describing functions in selected degrees of freedom. The describing functions give the relation between the provided input and the measured output of 
a system in terms of a magnitude and phase distortion at the input frequencies.

The measured describing functions are strictly speaking only valid at the measurement frequency and amplitude, as all motion systems are non-linear to a certain degree. ${ }^{12}$ However, for systems that are only slightly non-linear, the describing functions approximately match the transfer functions of a linear system. Thus, the describing function can be considered a linear description of the system dynamics.

The measurement signals were two multi-sine signals that contained five and six frequency/amplitude pairs, respectively. These have been used in previous research in determining the describing functions of the MPI Stewart platform. ${ }^{11}$ Frequencies up to $7.1 \mathrm{~Hz}$ were used and amplitudes up to $0.2 \mathrm{~m} / \mathrm{s}^{2}$ for the translational degrees of freedom, and up to $1.0 \mathrm{rad} / \mathrm{s}^{2}$ for the rotational degrees of freedom.

The describing functions are represented in a Bode diagram that plots magnitude and phase as a function of frequency. In this way, important system characteristics such as resonance and time delay can easily be determined.

\section{B. Advani-Hosman criterion}

The Advani-Hosman criterion aims to objectively qualify and regulate the motion cueing performance of flight simulators. ${ }^{21,22}$ It encompasses the entire cueing system, which consists of the motion cueing algorithms, motion platform hardware and controllers, and the time delays. The criterion plots the magnitude and phase of the cueing system response in a Nichols diagram, which is similar to a Bode diagram for the describing functions discussed above. However, the frequencies associated with the magnitude and phase are not considered, as these reveal the dynamic characteristics of the cueing system, which are often considered proprietary to the simulator manufacturer. ${ }^{22}$ On the other hand, the criterion defines performance bounds that are currently in an initial revision. These will be refined in the future. ${ }^{22}$

In this research, the criterion is used to compare the dynamic response of the MPI Stewart platform and the SRS without motion cueing filters. The describing function measurements are easily transformed into the criterion and compared with its bounds.

\section{MPI Stewart platform model analysis}

For simulation on the SIMONA Research Simulator, the model of the MPI Stewart platform model given in Section III was implemented in DUECA, the real-time software framework used for controlling the simulator. However, several difficulties were encountered, which led to further analysis of the platform model.

The describing functions of the complete model, given in Fig. 2, were determined by simulating the model response to the describing function measurement signals. Thus, the describing functions were determined for the complete system, including the platform controller, for all degrees of freedom.

The results of this analysis are given in Fig. 5. As is clearly visible, the dynamics of the platform model are mainly governed by a flat one-to-one response with a time delay. For each degree of freedom a first order model with a time delay was fit to the response. The time delay was found to be $0.035 \mathrm{~s}$ in all degrees of freedom. This corresponds to the value used in the simulations. The time constants for the first order models, $t_{b}$, are given in Table 4 and were very comparable for the translational degrees of freedom and for the rotational degrees of freedom. They represent break frequencies high above the frequency range of interest for the MPI Stewart platform model.

Given that the response of the MPI Stewart platform model almost exclusively equals a gain of one for the frequency range of interest, it was decided to not include the entire model in the final simulations of the MPI Stewart platform on the SRS. The response of the MPI Stewart platform is predominantly determined by the platform filters implemented by the platform manufacturer, see Eq. (1), and thus it suffices to only integrate these to got a reliable representation of the MPI Stewart platform on the SRS. Additionally, a time delay was implemented to match the time delay of the SRS to a delay of $0.035 \mathrm{~ms}$ found for the MPI Stewart platform.

Table 4: Parameters for the model describing functions.

\begin{tabular}{ccccccc}
\hline \hline & $\mathrm{x}$ & $\mathrm{y}$ & $\mathrm{z}$ & $\phi$ & $\theta$ & $\psi$ \\
\hline$t_{b}, s$ & 0.00560 & 0.00561 & 0.00081 & 0.01243 & 0.01242 & 0.01398 \\
\hline \hline
\end{tabular}


Thus, the response of the MPI Stewart platform is modelled with the following equation in every degree of freedom:

$$
H_{\text {model }}=\frac{1}{\left(1+\frac{1}{2 \pi f_{b}} s\right)^{2}} \cdot e^{-\tau s},
$$

where $f_{b}$ is the break frequency and $\tau$ the time delay of the model.
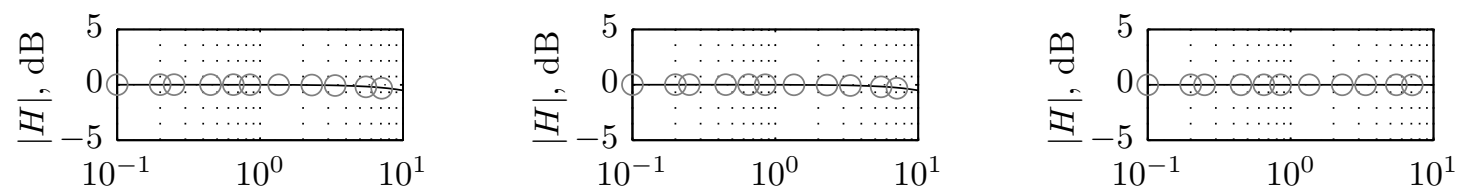

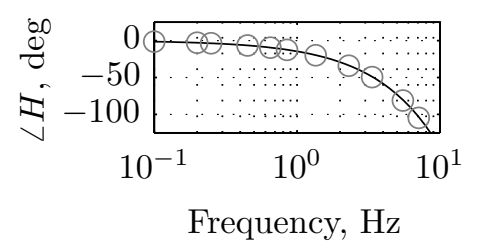

(a) Surge.
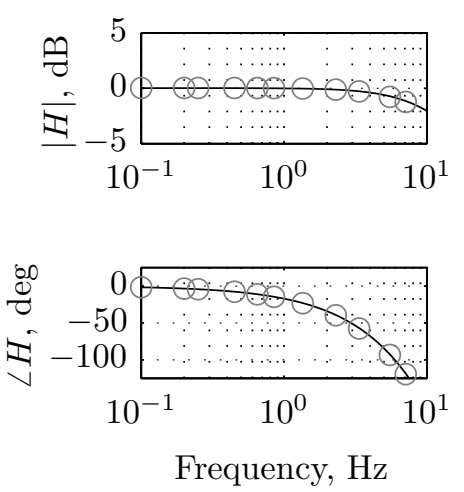

(d) Roll.

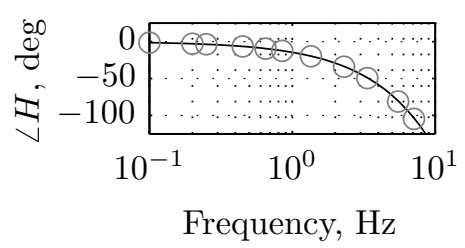

(b) Sway.
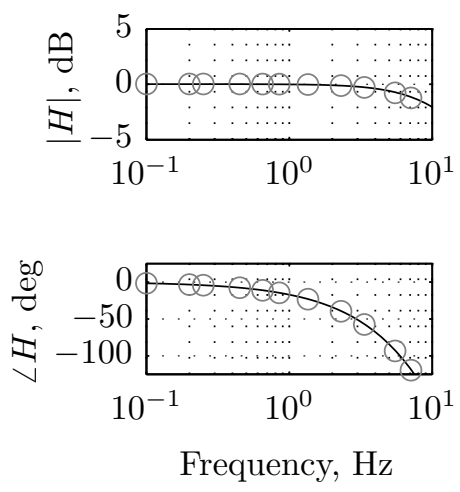

(e) Pitch.

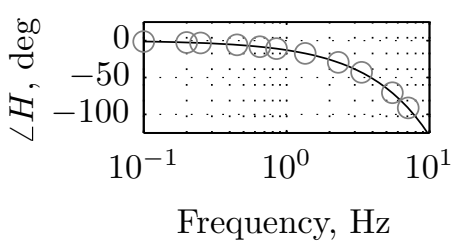

(c) Heave.
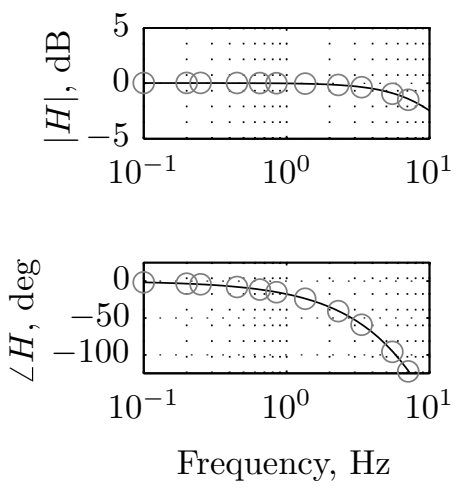

(f) Yaw.

Figure 5: MPI Stewart platform model describing functions.

\section{SRS measurements and simulation results}

The reduced model of the MPI Stewart platform given in Eq. (9) was implemented on the SRS. As validation, describing function measurements as described before were performed in heave and pitch, as these degrees of freedom will be used in subsequent closed-loop control experiments. In this case, the describing functions include the motion system and simulator cab dynamics, and the time delays introduced by the motion control computer and simulation software. No motion cueing filters were used.

Three values were used for the break frequency $f_{b}: 1 \mathrm{~Hz}, 5 \mathrm{~Hz}$, and $10 \mathrm{~Hz}$. These values cover the entire operating range of the MPI Stewart platform, where $1 \mathrm{~Hz}$ is equal to the default platform filter break frequency implemented by the manufacturer. Additionally, the describing function measurements were performed without the model, revealing the full performance of the SRS. Two values for the time delay $\tau$ were tested. No additional time delay was introduced $(\tau=0)$ to reveal the time delays in the SRS. To check the model implementation, $\tau$ was taken $0.035 \mathrm{~s}$, which is equal to the time delay found in measurements on the MPI Stewart platform. ${ }^{13}$

The SRS motion was measured with two Inertial Measurement Units (IMU). The SRS is equipped with an ISIS IMU (Rev. C) from Inertial Science Inc., an older MEMS-based device. ${ }^{23}$ Data from this IMU 

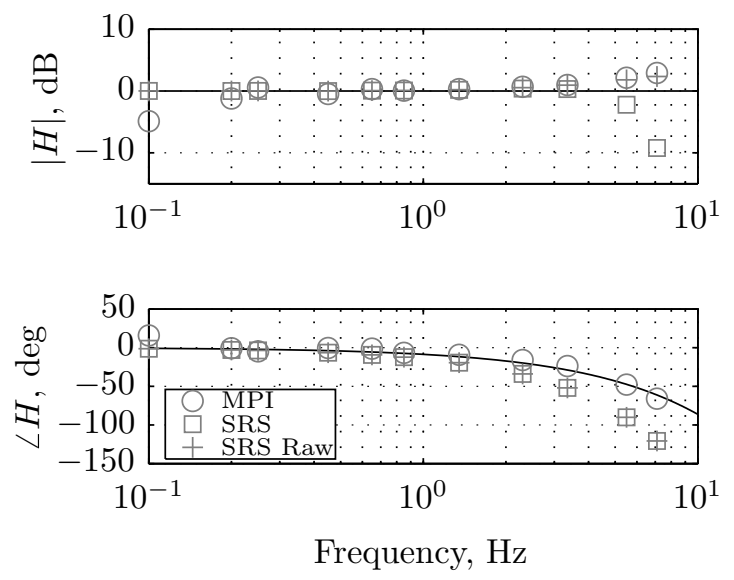

(a) Heave.
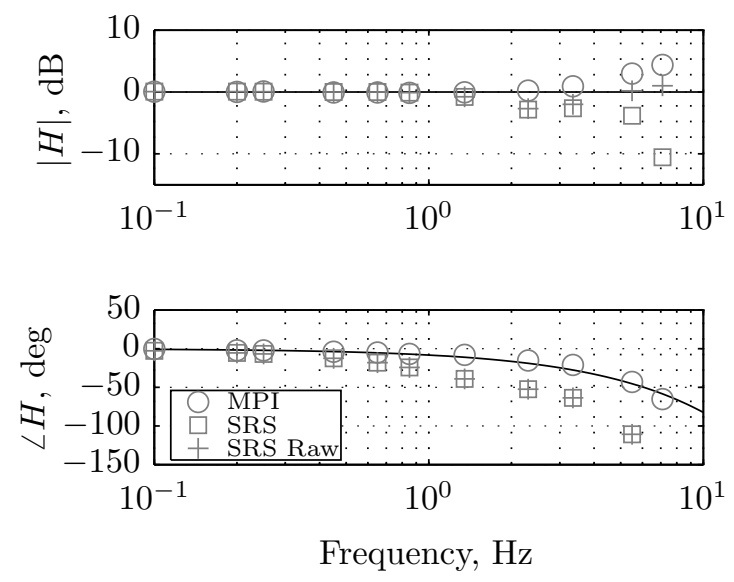

(b) Pitch.

Figure 6: Describing functions measured with different IMUs.

are processed with a filter with a cut-off frequency of $5 \mathrm{~Hz}$, but also raw data from the device are logged. Additionally, the ADIS16355 IMU (MPI IMU) from Analog Devices, Inc. was integrated in the SRS for the validation. Previously, the performance measurements on the MPI Stewart platform were performed with this IMU. ${ }^{11}$

The results from the describing function measurements without the MPI Stewart platform model are given in Fig. 6. For heave, it is clear that the magnitude of the response from the SRS IMU is attenuated at higher frequencies. This is due to the characteristics of the filters for signals from the SRS IMU. When taking the raw signal from the SRS IMU, the slight resonance of the SRS at higher frequencies is picked up correctly and is similar to the response measured with the MPI IMU. For the lowest frequency, the MPI IMU shows a deviation in magnitude which is attributed to measurement noise. For the measurement in pitch, Fig. 6b shows that the response from the SRS IMU is attenuated above $1 \mathrm{~Hz}$, probably due to internal filtering. The MPI IMU shows a similar pattern as was found in heave, with slight resonance for the higher frequencies.

The phase response of the SRS shows that the time delay measured with the MPI IMU is significantly lower than the time delay measured with the SRS IMU. The MPI IMU is from a newer generation of MEMSbased IMUs, and probably uses less internal filtering and processing. The MPI IMU is considered to yield more representative data, as the measured magnitude and phase in the describing function measurements are more comparable to previous measurements with a high-performance IMU and measurements of leg lengths on the SRS than data from the SRS IMU.

In Fig. 7, the describing function measurements with the MPI Stewart platform model are given. The data were measured with the MPI IMU. As can be seen in Fig. 7a, the magnitude of the SRS response in heave and pitch follows the analytical model well, although the resonance at the highest frequencies introduces some discrepancies in both degrees of freedom.

With the phase of the SRS response, the time delay in the system can be estimated by setting $f_{b}$ in Eq. (9) to the simulated value and fitting $\tau$. The results for the SRS and the different break frequencies of the MPI Stewart platform model are given in Table 5.

For the SRS, time delays of approximately $24 \mathrm{~ms}$ were found with the MPI IMU for heave and pitch. When the MPI Stewart platform model is simulated, a lower time delay of $19 \mathrm{~ms}$ is found for break frequencies of 5 and $10 \mathrm{~Hz}$. With the SRS IMU, similar results are found, although this IMU has an additional delay of approximately $22 \mathrm{~ms}$.

For the model with a break frequency of $1 \mathrm{~Hz}$, a time delay of approximately $13 \mathrm{~ms}$ is found with the MPI IMU. This value is much lower than the time delays found with the models with higher break frequencies. On the contrary, the time delay found with the SRS IMU is similar to time delays of the models with higher break frequencies, as is shown in Table 5. These discrepancies are not related to the implementation of the MPI Stewart platform model, as in measurements with $\tau=0.035$ an additional delay of $35 \mathrm{~ms}$ is found. A 

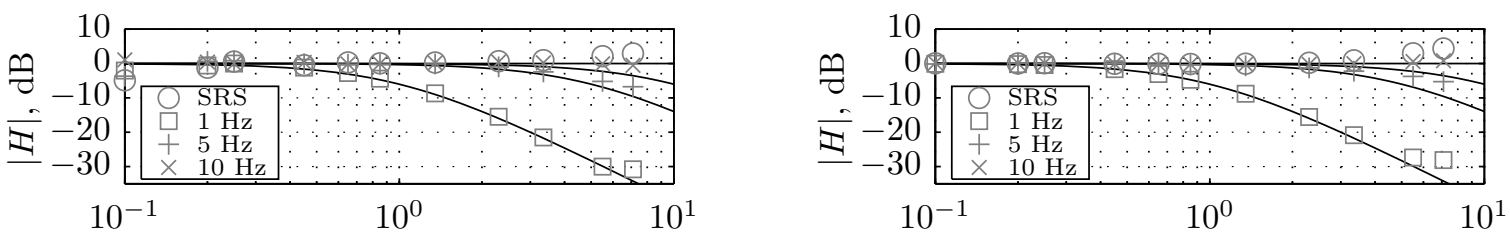

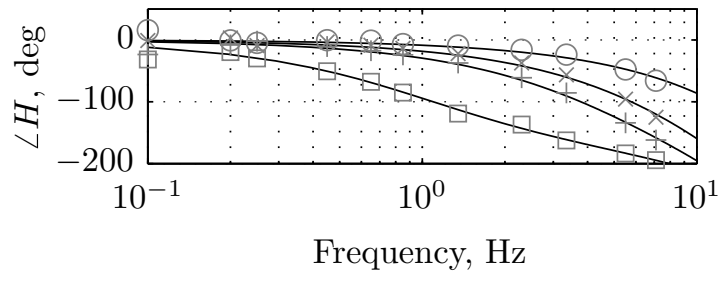

(a) Heave.

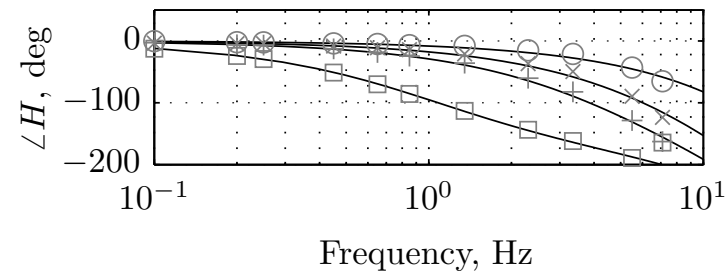

(b) Pitch.

Figure 7: Measured describing functions for different platform filters.

possible reason for these findings could be that the most important data for the time delay estimation are the measured response at higher frequencies. However, for the $1 \mathrm{~Hz}$ filter, these measurements are unreliable due to the low gains of the filter at higher frequencies. For the MPI IMU, the measurements are probably affected by a low signal-to-noise ratio.

The measured describing functions are given in a Nichols plot with the Advani-Hosman criterion in Fig. 8. Note that the describing functions do not include the influence of motion cueing filters, which were not used in these measurements. These would decrease correspondence with the criterion.

It is clear from the figure that it is difficult to determine the response for heave at the lowest frequencies. However, the SRS shows good correspondence with the criterion up to $3.35 \mathrm{~Hz}(21 \mathrm{rad} / \mathrm{s})$. This is well above the frequency range of $2-5 \mathrm{rad} / \mathrm{s}$ where the pilot-vehicle system crossover frequency is expected to be for closed-loop control tasks. ${ }^{24}$

On the other hand, when the break frequency of the MPI Stewart platform model decreases, the system response becomes positioned further from the favourable region of the criterion. For the break frequency of $1 \mathrm{~Hz}$, the system response falls outside the criterion for frequencies larger than $0.5 \mathrm{~Hz}(\approx 3 \mathrm{rad} / \mathrm{s})$. Thus, the default platform filters of the MPI Stewart platform have a large effect on pilot control in closed-loop tasks.

Table 5: Time delays measured with different IMUs for different platform filters.

\begin{tabular}{lcccc}
\hline \hline & \multicolumn{2}{c}{ heave } & \multicolumn{2}{c}{ pitch } \\
MPI IMU & $\tau=0.0$ & $\tau=0.035$ & $\tau=0.0$ & $\tau=0.035$ \\
\hline SRS & 0.024 & 0.059 & 0.023 & 0.058 \\
$1 \mathrm{~Hz}$ & 0.012 & 0.048 & 0.014 & 0.051 \\
$5 \mathrm{~Hz}$ & 0.019 & 0.053 & 0.018 & 0.052 \\
$10 \mathrm{~Hz}$ & 0.019 & 0.053 & 0.018 & 0.053 \\
\hline SRS IMU & & & & \\
\hline SRS & 0.046 & 0.081 & 0.059 & 0.094 \\
$1 \mathrm{~Hz}$ & 0.042 & 0.077 & 0.054 & 0.089 \\
$5 \mathrm{~Hz}$ & 0.041 & 0.076 & 0.054 & 0.089 \\
$10 \mathrm{~Hz}$ & 0.041 & 0.076 & 0.054 & 0.089 \\
\hline \hline
\end{tabular}




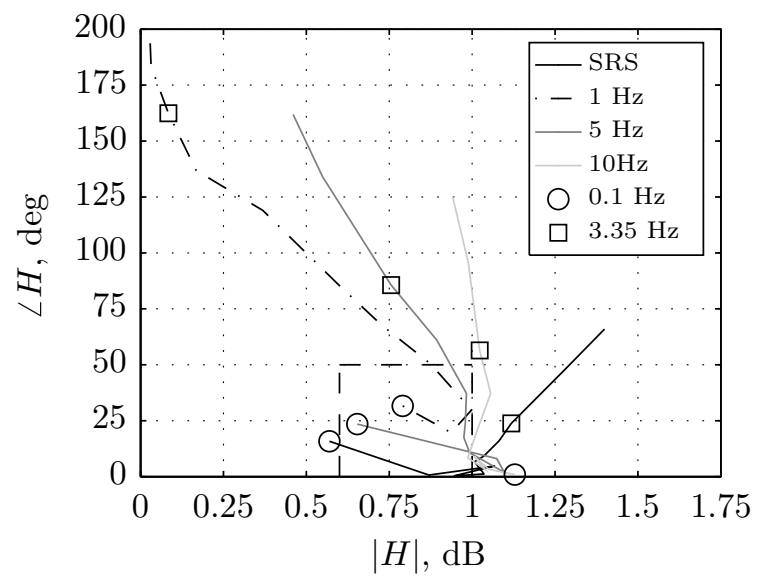

(a) Heave.

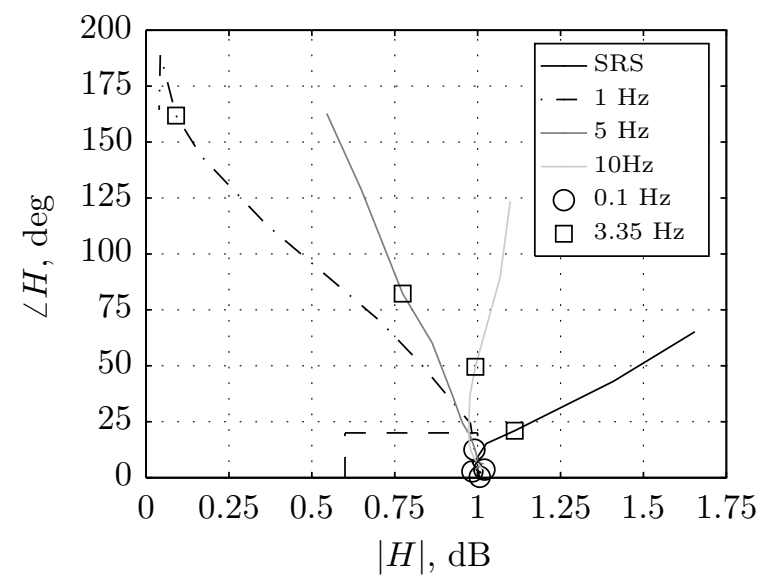

(b) Pitch.

Figure 8: Measured describing functions in relation to the Advani-Hosman criterion.

\section{Conclusion}

Describing function measurements were performed to validate a model of the MPI Stewart platform. In these measurements, a lower time delay was measured for the SIMONA Research Simulator with a new IMU in comparison with the standard SRS IMU. Analysis also revealed a resonance peak at high frequencies. The implementation of the MPI Stewart platform model on the SRS has been validated and can thus be used for subsequent experiments on human perception and control behaviour in active closed-loop control tasks. The resonance of the SRS could be corrected with additional filters, but is not expected to influence the experiments as the resonance frequency range is well above the frequency range of human control inputs.

\section{Acknowledgements}

Heinrich H. Bülthoff was supported by the World Class University program through the National Research Foundation of Korea funded by the Ministry of Education, Science and Technology (R31-2008-000-10008-0).

\section{References}

${ }^{1}$ Bürki-Cohen, J., Soja, N. N., and Longridge, T., "Simulator Platform Motion - The Need Revisited," The International Journal of Aviation Psychology, Vol. 8, No. 3, 1998, pp. 293-317.

${ }^{2}$ Go, T. H., Bürki-Cohen, J., Chung, W. W. Y., Schroeder, J. A., Saillant, G., Jacobs, S., and Longridge, T., "The Effects of Enhanced Hexapod Motion on Airline Pilot Recurrent Training and Evaluation," Proceedings of the AIAA Modeling and Simulation Technologies Conference and Exhibit, Austin (TX), No. AIAA-2003-5678, 11-14 Aug. 2003.

${ }^{3}$ Stapleford, R. L., Peters, R. A., and Alex, F. R., "Experiments and a Model for Pilot Dynamics with Visual and Motion Inputs," NASA Contractor Report NASA CR-1325, NASA, 1969.

${ }^{4}$ Zaal, P. M. T., Pool, D. M., de Bruin, J., Mulder, M., and van Paassen, M. M., "Use of Pitch and Heave Motion Cues in a Pitch Control Task," Journal of Guidance, Control, and Dynamics, Vol. 32, No. 2, March-April 2009, pp. 366-377.

${ }^{5}$ Pool, D. M., Mulder, M., van Paassen, M. M., and Vaart, J. C. v., "Effects of Peripheral Visual and Physical Motion Cues in Roll-Axis Tracking Tasks," Journal of Guidance, Control, and Dynamics, Vol. 31, No. 6, Nov.-Dec. 2008, pp. $1608-1622$.

${ }^{6}$ Zaal, P. M. T., Nieuwenhuizen, F. M., Mulder, M., and van Paassen, M. M., "Perception of Visual and Motion Cues During Control of Self-Motion in Optic Flow Environments," Proceedings of the AIAA Modeling and Simulation Technologies Conference and Exhibit, Keystone (CO), No. AIAA-2006-6627, 21-24Aug. 2006.

${ }^{7}$ Pool, D. M., Zaal, P. M. T., van Paassen, M. M., and Mulder, M., "Effects of Heave Washout Settings in Aircraft Pitch Disturbance Rejection," Journal of Guidance, Control, and Dynamics, Vol. 33, No. 1, Jan.-Feb. 2010, pp. $29-41$.

${ }^{8}$ Nieuwenhuizen, F. M., Zaal, P. M. T., Teufel, H. J., Mulder, M., and Bülthoff, H. H., "The Effect of Simulator Motion on Pilot Control Behaviour for Agile and Inert Helicopter Dynamics," Proceedings of the 35th European Rotorcraft Forum, Hamburg, Germany, 22-25 2009.

${ }^{9}$ Nieuwenhuizen, F. M., Zaal, P. M. T., Mulder, M., van Paassen, M. M., and Mulder, J. A., "Modeling Human Multichannel Perception and Control Using Linear Time-Invariant Models," Journal of Guidance, Control, and Dynamics, Vol. 31, No. 4, July-Aug. 2008, pp. 999-1013. 
${ }^{10}$ Zaal, P. M. T., Pool, D. M., Chu, Q. P., van Paassen, M. M., Mulder, M., and Mulder, J. A., "Modeling Human Multimodal Perception and Control Using Genetic Maximum Likelihood Estimation," Journal of Guidance, Control, and Dynamics, Vol. 32, No. 4, July-Aug. 2009, pp. 1089-1099.

${ }^{11}$ Nieuwenhuizen, F. M., Beykirch, K. A., Mulder, M., van Paassen, M. M., Bonten, J. L. G., and Bülthoff, H. H., "Performance Measurements on the MPI Stewart Platform," Proceedings of the AIAA Modeling and Simulation Technologies Conference and Exhibit, Honolulu (HI), No. AIAA-2008-6531, 18-21Aug. 2008.

${ }^{12}$ Lean, D. and Gerlach, O. H., "AGARD Advisory Report No. 144: Dynamics Characteristics of Flight Simulator Motion Systems," Tech. Rep. AGARD-AR-144, North Atlantic Treaty Organization, Advisory Group for Aerospace Research and Development, 1979.

${ }^{13}$ Nieuwenhuizen, F. M., van Paassen, M. M., Mulder, M., Beykirch, K., and Bülthoff, H. H., "Towards Simulating a Mid-size Stewart Platform on a Large Hexapod Simulator," Proceedings of the AIAA Modeling and Simulation Technologies Conference and Exhibit, Chicago (IL), No. AIAA-2009-5917, 10-13Aug. 2009.

${ }^{14}$ Stewart, D., "A Platform With Six Degrees of Freedom," Institution of Mechanical Engineers, Proceedings 1965-1966, Vol. 180 Part I, 1966, pp. 371-378.

${ }^{15}$ Berkouwer, W. R., Stroosma, O., van Paassen, M. M., Mulder, M., and Mulder, J. A., "Measuring the Performance of the SIMONA Research Simulator's Motion System," Proceedings of the AIAA Modeling and Simulation Technologies Conference and Exhibit, San Francisco (CA), No. AIAA-2005-6504, 15-18 Aug. 2005.

${ }^{16}$ van Paassen, M. M., Stroosma, O., and Delatour, J., "DUECA - Data-Driven Activation in Distributed Real-Time Computation," Proceedings of the AIAA Modeling and Simulation Technologies Conference and Exhibit, Denver (CO), No. AIAA-2000-4503, 14-17 Aug. 2000.

${ }^{17}$ Koekebakker, S. H., Model Based Control of a Flight Simulator Motion System, Doctoral dissertation, Faculty of Aerospace Engineering, Delft University of Technology, 2001.

${ }^{18}$ Advani, S. K., The Kinematic Design of Flight Simulator Motion-Bases, Doctoral dissertation, Faculty of Aerospace Engineering, Delft University of Technology, 1998.

${ }^{19}$ Harib, K. and Srinivasan, K., "Kinematic and dynamic analysis of Stewart platform-based machine tool structures," Robotica, Vol. 21, 2003, pp. 541-554.

${ }^{20}$ Tischler, M. B. and Remple, R. K., Aircraft and Rotorcraft System Identification, American Institute of Aeronautics and Astronautics, Inc., 1801 Alexander Bell Drive, Reston, VA, 2006.

${ }^{21}$ Advani, S. K. and Hosman, R. J. A. W., "Revising Civil Simulator Standards - An Opportunity for Technological Pull," Proceedings of the AIAA Modeling and Simulation Technologies Conference and Exhibit, Keystone (CO), No. AIAA-2006-6248, 21-24 Aug. 2006.

${ }^{22}$ Advani, S. K., Hosman, R. J. A. W., and Potter, M., "Objective Motion Fidelity Qualification in Flight Training Simulators," Proceedings of the AIAA Modeling and Simulation Technologies Conference and Exhibit, Hilton Head (SC), No. AIAA-2007-6802, 20-23 Aug. 2007.

${ }^{23}$ Pool, D. M., Chu, Q. P., Mulder, M., and van Paassen, M. M., "Optimal Reconstruction of Flight Simulator Motion Cues using Extended Kalman Filtering," Proceedings of the AIAA Modeling and Simulation Technologies Conference and Exhibit, Honolulu (HI), No. AIAA-2008-6539, 18-21 Aug. 2008.

${ }^{24}$ McRuer, D. T. and Jex, H. R., "A Review of Quasi-Linear Pilot Models," IEEE Transactions on Human Factors in Electronics, Vol. HFE-8, No. 3, 1967, pp. 231-249. 